Copyright ( 2020 University of Bucharest Printed in Romania. All rights reserved

ISSN print: $1224-5984$

ISSN online: $2248-3942$
Rom Biotechnol Lett. 2020; 25(5): 2008-2017 doi: $10.25083 / \mathrm{rbl} / 25.5 / 2008.2017$

Received for publication, November, 3, 2019

Accepted, December, 29, 2019

\title{
Microscopic study of endodontic ledges on extracted teeth
}

\section{IOANA SUCIU ${ }^{1}$, MIHAI CIOCARDEL ${ }^{2}$, BOGDAN DIMITRIU ${ }^{1}$, ECATERINA IONESCU ${ }^{1}$, MIHAELA CHIRILA ${ }^{1}$, ELINA TEODORESCU ${ }^{1}$,MILICESCU STEFAN ${ }^{1}$, ANCA DUMITRIU ${ }^{1}$, DANA BODNAR ${ }^{1}$,GRATIELA GRADISTEANU PIRCALABIORU ${ }^{3}$, STANA PAUNICA ${ }^{1}$, GEORGIANA CROITORU ${ }^{1}$, RUXANDRA BARTOK ${ }^{1}$, OANA AMZA ${ }^{1}$}

${ }^{1}$ Faculty of Dental Medicine, "Carol Davila" University of Medicine and Pharmacy, Bucharest, Romania ${ }^{2}$ Department of Geology Petroleum-Gas University of Ploiesti, Ploiesti, Romania

${ }^{3}$ Research Institute of University of Bucharest (ICUB), Bucharest, Romania

\begin{abstract}
Endodontic ledges are one of the major problems encountered in endodontic treatments performed on curved roots. The existence of a ledge hinders the achievement of an adequately shaped canal preparation and this can trigger incomplete instrumentation and disinfection of the root canal system as well as incomplete canal filling. The present study aimed to highlight the microscopic characteristics of endodontic ledges formed during in vivo treatments, as well to analyse their relationship with the rest of the root canal left inaccessible during mechanical canal treatment. We also aimed to correlate the appearance of the ledges with the degree of curvature of the roots on which they were found. An attempt was made to introduce a measure to reflect the severity of the ledge depending on how much the endodontic instrument 'dug' into the root dentin deviating from the direction of the root canal. We believe that this would be related to the ease with which a ledge for a possible resumption of the endodontic treatment could be exceeded and eliminated.
\end{abstract}

Keywords Endodontic ledges, endodontic instruments, working length.

To cite this article: SUCIU I, CIOCARDEL M, DIMITRIU B, IONESCU E, CHIRILA M, TEODORESCU E, STEFAN M, DUMITRIU A, BODNAR D, PIRCALABIORU GG, PAUNICA S, CROITORU G, BARTOK R, AMZA O. Microscopic study of endodontic ledges on extracted teeth. Rom Biotechnol Lett. 2020; 25(5): 2008-2017. DOI: $10.25083 / \mathrm{rbl} / 25.5 / 2008.2017$

\footnotetext{
*Corresponding author: MIHAELA CHIRILA, Faculty of Dental Medicine, "Carol Davila" University of Medicine and Pharmacy, Bucharest, Romania

E-mail: mihaela.chirila@umfcd.ro
} 


\section{Introduction}

Among the complications most commonly encountered during root canal instrumentation is a deviation from the original canal curvature with no communication with the periodontal ligament, leading to a procedural error also known as ledge formation. The existence of a ledge impairs the achievement of an adequately shaped canal preparation and this can trigger incomplete instrumentation and disinfection of the root canal system and also incomplete filling of the canal. (JAFARZADEH \& al [1], SCARLATESCU $\&$ al [2], The present study aimed to highlight the microscopic characteristics of endodontic ledges formed during in vivo treatments, as well to highlight their relationship with the rest of the root canal left inaccessible during the mechanical canal treatment. We also aimed to correlate the appearance of the ledges with the degree of curvature of the roots on which they were found. An attempt was made to introduce a measure to reflect the severity of the ledge depending on how much the endodontic instrument 'dug' into the root dentin deviating from the direction of the root canal. We believe that this would be related to the ease with which a ledge for a possible resumption of the endodontic treatment could be exceeded and eliminated.

Endodontic ledges are one of the major problems encountered in endodontic treatments performed on curved roots. Endodontic ledges have been defined in several ways: i) a deviation from the original curvature of the canal without communication with the periodontal ligament (JAFARZADEH \& al [1]); ii) an irregular iatrogenic product, having the shape of a platform in the root canal wall, which makes access to the apex in a previously permeable canal difficult or even impossible (HÜLSMANN $\&$ al [3]). It is currently appreciated that the formation of ledges is favoured by: the use of rigid endodontic instruments, the large curvature of the root canals, the lack of the initial negotiation of the root curves with thin canal files $(.06, .08,0.10)$, incorrect access cavity, erroneous determination of the working length (KAPALAS \& al [4]).

A less discussed fact is the favouring of the formation of ledges by the presence of intracanal calcifications that are difficult to dissolve and that, by opposing a resistance to the advancement of the canal files similar to that of ordinary dentin, forces the instrument to 'dig' out of the real direction of the root canal (LAMBRIANIDIS \& al [5]). The formation of a ledge can prevent the completion of the endodontic treatment by the inability to access with endodontic instruments the portion of the canal located from the ledge site to the apical. The presence of ledges is often associated with incomplete root canal fillings, which can be relatively easily detected radiologically. These are often reasons for the resumption of endodontic treatments by endodontic specialists, although in the case of asymptomatic teeth and without paraclinical signs of periapical pathology, the obligation to resume treatment is debatable (ELEFTHERIADIS \& al [6]).

\section{Materials and Methods}

For this microscopic study of the endodontic ledges formed during the endodontic treatments, it was necessary to obtain some teeth with endodontic ledges and therefore with unsuccessful canal treatments, following some dental extractions. The selection of cases and their follow-up was extremely long and difficult. The endodontic ledges cannot be highlighted directly on patients' dental radiographs, but their presence has been deduced by identifying incomplete canal fillings, up to levels where there is a significant change in the direction of the root canal. Teeth with incomplete canal fillings were extracted due to severe damage to the marginal periodontium, associated with high mobility or due to the presence of surgically unscoopable periapical cysts than through the postextractional alveolus. Extraction due to damage to the marginal periodontium was performed when the loss of bone support due to typical chronic deep periodontitis (slowly progressive) was very advanced. The patients' consent was obtained for the use of the teeth extracted in the study and the periodontologists from the faculty were consulted regarding the treatment of periodontal disease and the indication for extraction.

Obviously, a large part of the cases pursued could be treated periodically with good or acceptable results, so that the extraction of teeth identified with incomplete fillings was not achieved. In such cases, after the main stage of periodontal treatment, the endodontic treatment was also resumed in the university clinic. The good results of periodontal treatments in some cases were just one of the reasons why the teeth on which the study could be based were few. Other factors that significantly reduced the number of teeth with microscopically studied ledged were: (1) lack of continued presentation of patients to periodontal treatments after the diagnosis of periodontal disease, as well as the presence of incomplete root canal treatments have been established; (2) lack of identification of ledges or lack of their capture in microscopic investigations, although canal fillings were incomplete; (3) unwanted destruction of the ledge portions when preparing microscopic preparations.

An exception to the tooth included in the study is a situation in which a retreatment was performed for an extensive and persistent periapical lesion, near a root that had a complete canal filling. The patient accepted outside our clinic, a retreatment, which was unsuccessful, meaning that the access to apical constriction was not successful. The patient was informed that 'an obstacle in the root prevented access to remove the old filling'. In this case, an endodontic ledge was actually created when the treatment was resumed. This case will be presented in more detail below.

After tooth extraction and taking measures to prevent microbial contamination, in vitro coronary fillings were removed with turbine and diamond burs. After the removal of the coronal fillings, the entrance places in the incompletely filled root canals were spotted as accurately as possible and where possible, through a strong transillumination, the direction of the canal filling in the root and the place where the filling ends (presumably ledge location) were also 
spotted. Sometimes post-extraction radiographs were also taken. Based on these observations, which were not always sufficiently precise, some very fine markings were made on the external root surfaces in order to constitute a guide for performing the root sectioning.

The section of the roots along them was performed with a microtome (LEIKA RM 2255 WETZLAR GERMANY) following as much as possible the capture of the ledge. It was assumed to exist at the site where the incomplete canal filling ends (to the apical, in a location located towards coronal relative to the apical constriction). The obtained section surfaces, in many cases, did not capture the root canal from the respective root along its entire length, due to its curves in several planes. The incomplete canal filling was removed after this first sectioning without acting on root fragments with metal tools. Only plastic toothpicks were used so as not to alter the walls of the root canals. The filling of the uninstrumented parts of the root canals (of their portions located apically to the place supposed to have the ledge) was not followed and was not removed with instruments. However, irrigation with pressure distilled water was performed in several steps on the section surfaces. This, however, led to the partial or sometimes total removal of residues present on the uninstrumented portions of the root canals. Exceptions were some dentinal detritus plugs or areas with dentinal detritus. This has facilitated for us their microscopic highlighting, which we do not consider unimportant.

The first microscopic investigations on the obtained root halves were made with a Leica EZ4 HD stereomicroscope (Switzerland), equipped with a photodigital camera. As previously mentioned, no ledge was detected in the case of some roots. In these cases, the reason why the canal filling was not complete remains debatable. Most likely, based on the clinical experience of the authors, the reasons may be erroneous determination of the working length or incorrect access cavity. The root fragments on which no ledges were identified and implicitly the respective teeth were excluded from the study.

In the end, we were left with a number of 11 roots with relatively clear ledges and which were subjected to further study. The images obtained with the stereomicroscope were made in the order of magnification of $8 x$ or $10 x$ and allow both the identification of the ledges (without details) and the observation of some general images on the root canals (on the portions on which they were captured). In one case, which will be presented below, a false path was also observed.

The root fragments on which ledges were identified were resected with the microtome so as to obtain $1 \mathrm{~mm}$ thick slices. The new section surfaces were made in parallel with the original ones. The aim was to obtain these slices to facilitate the microscopic study in light transmitted by a polarizing microscope. The research microscope used was a Leica DM EP model (Switzerland) equipped with a special photodigital camera dedicated to microscopy (Leica DMC 4500). With this microscope and the attached camera, additional detailed images were obtained on the ledges and sometimes on other features identified on the studied roots (for example: apical transposition aspects). At least two digital photographs were taken with this microscope: one at the lower magnification order $(30 \mathrm{x})$ and one at the magnification order $40 \mathrm{x}$ or $100 \mathrm{x}$, depending on the case. Although transmitted light was often used, reflected light or a combination of transmitted light and reflected light was also used for a few situations. The choice of the lighting variant was chosen by the microscopist to best capture the aspects of interest, in particular, the ledge area.

On one of the microscopy images taken at higher degrees of magnification (40x or 100x), which we consider in this study a detailed image of the ledge, prepared portion of the adjacent root canal ledge and the unprepared portion of the root canal adjacent to the ledge were clearly delimited with colour dotted lines. The same detail image is presented and unmarked to facilitate the observation of the details that were covered by our markings.

In order to make the final interpretations possible, two parameters were appreciated: (1) curvature of the root on which the ledge was encountered; (2) a deviation, representing the distance from the central portion of the ledge to the axis of the root canal at the same depth level in the apical-coronal direction. We have introduced this parameter in order to express in the most relevant way what is the 'ledge gravity'.

Both parameters are schematically highlighted in Figure 1.

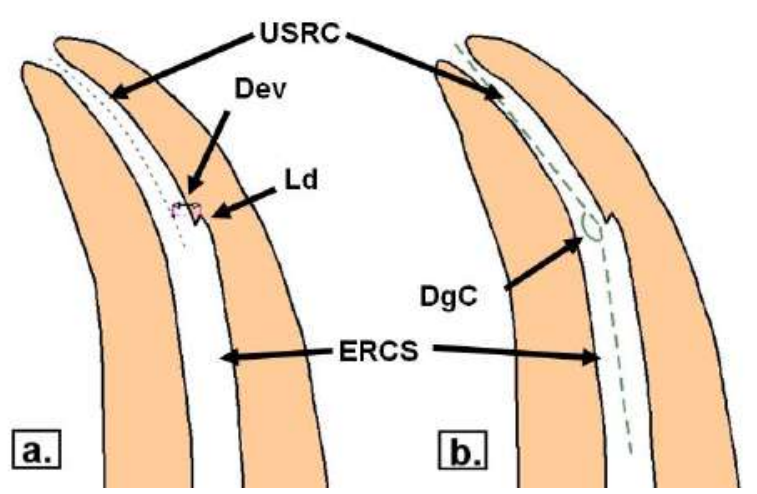

Figure 1. Illustrative diagram for the notions of deviation in the formation of a ledge (a) and degree of curvature of the root (b) Dev - deviation; Ld - ledge; DgC - degree of curvature; USRC - uninstrumented segment of the root canal; ERCS - enlarged root canal segment. 
The root curvature was expressed as an angle between a segment superimposed over the portion of the canal located coronal to the ledge (the one on which the root canal filling was found) and a segment superimposed over the uninstrumented portion of the canal (the one that is without filling or could not be instrumented at the retreatment) (Fig. 1a). Obviously, this way of expressing the curve is a simplification, but still the evaluation is easy and in a similar form it has been used by other authors (KHANNA $\&$ al [7]). This curvature was evaluated on one of the microscopic photographs, usually the stereomicroscopy one, because having the smallest magnification order, it completely captures the root on which the ledge was identified. In the case of some teeth, image collages were also made to obtain a complete image with the root. In such cases, an image capturing the coronal and radicular-coronal portion and an image with a root-apical portion of the tooth were joined (example e.g. Fig 2b). In order to obtain graphically the mentioned angle, the two segments were marked on the photodigital images. Some roots on which ledges have been found have another curvature located in a different plane than the one in which the main one is located. It was no longer appreciated graphically but was visually estimated on the extracted tooth before sectioning using a small plastic protractor. Usually this secondary curvature strictly affects the apical third of the root. Its expression was done using values every 5 degrees. No better accuracy could be provided. In order to determine more precisely this second curvature, it would have been necessary to section each root in two planes, which would have made it impossible to microscopically follow the root canals, since the second sectioning plane would have been relatively perpendicular to the first and would have had to pass through the middle of the root.

The deviation could be determined based on the calibration marks of the digital photographs taken with a stereomicroscope or microscope. However, this method of assessment involves larger errors than direct measurement with previously calibrated micrometric eyepieces. This second option was used. The distance from the middle of the ledge to the middle of the root canal located at the same level (in the same plane of cross section) was measured with the calibrated micrometric eyepiece (Fig. 1b).

\section{Results and Discussions}

In order to reveal the characteristics of the endodontic ledges and their relations with the rest of the root canal, we considered it necessary to present some concrete cases from those studied by us, namely some that we found to be more interesting. For this we used for each case a set of relevant images. Given the great diversity of the situations encountered, it is obvious that a common general comment cannot be made for all the cases investigated. A totally atypical example is case 2 below. We could not include in this article all the images obtained during the study.
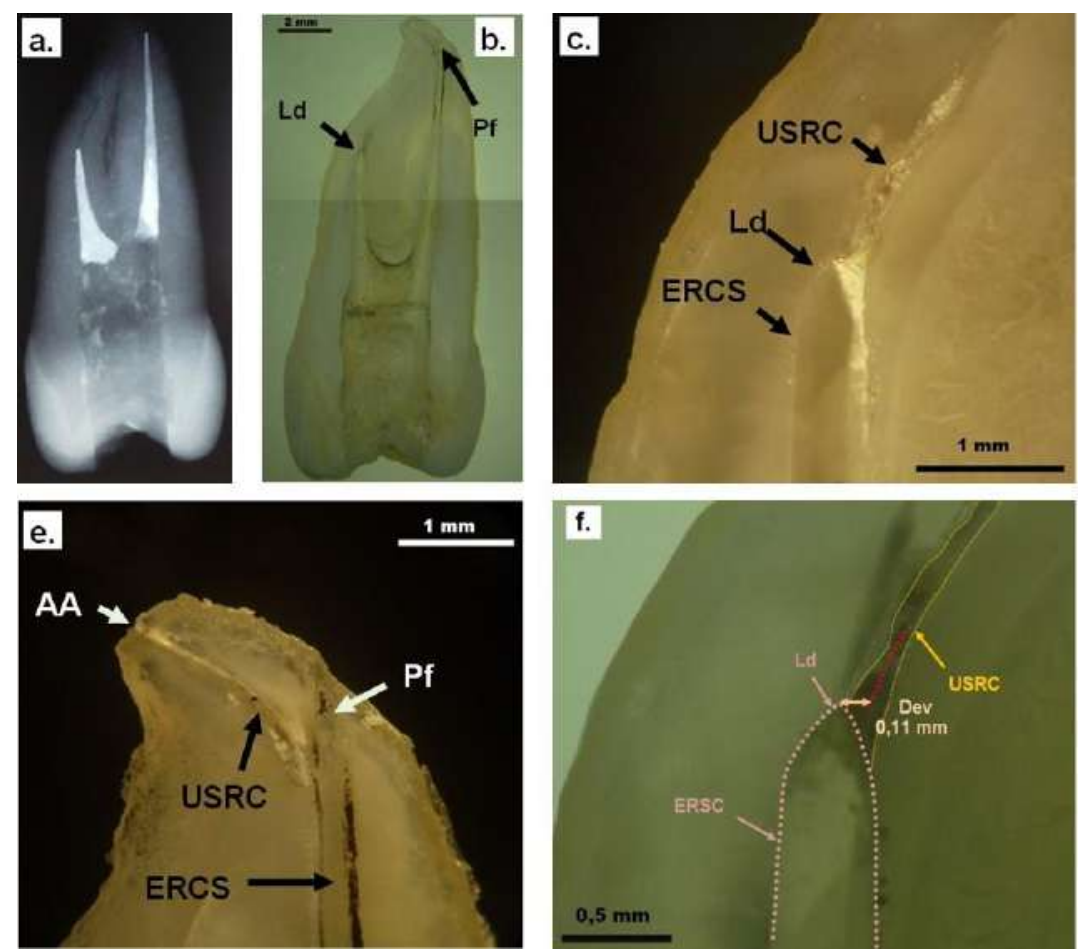
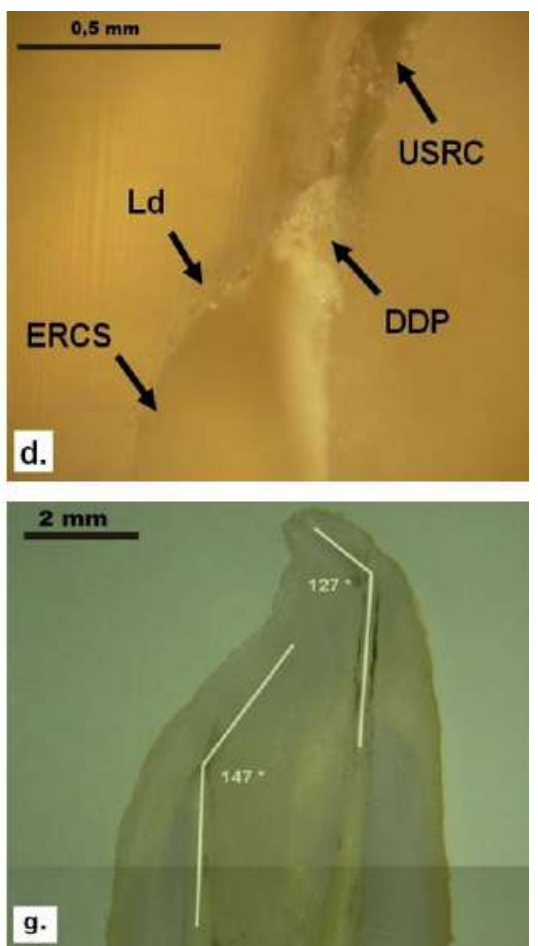

Figure 2. First maxillary premolar (1.4) with a ledge present on the palatal root and perforation present on the buccal rootCase 1 - Tooth Sample 01. a) post-extraction radiological aspect; b) Transmitted light stereomicroscopy image (8x); c) Light microscopy image reflected with the endodontic ledge (30x); d) Detail image of light microscopy reflected with the ledge $(100 \mathrm{x})$; e) Light microscopy image reflected with the false path (perforation) produced in the apical area of the root by improper instrumentation of the buccal canal (30x); f) Light microscopy image transmitted with the ledge, having outlined the main aspects of interest (30x); g) Half from the apex of the stereomicroscopy image b., used for marking and calculating the angles of curvature of the root canals. Abbreviations used: Ledge = Ld; Perforation = Pf; Enlarged root canal segment $=$ ERCS; Uninstrumented segment of the root canal $=$ USRC; Anatomic apex = AA; Dev=deviation. 
Case no. 1. On the first upper premolar we noticed the existence of a ledge on the palatal canal and a ledge with a false path on the buccal canal. In the case of this first maxillary premolar, we found a false ledge association. It can be said that, due to the fact that the usual radiographs are a projection in the buccal-oral plane, the perforation would not have been noticed radiologically. It should be noted, however, that neither in mesio-distal projection, on postextractional radiography, made for research purposes, the false path is obvious. The filling of the buccal canal seems complete and correct. The uninstrumented portion of the buccal canal is not radiologically evident. Only the microscopic study and the images obtained in it reflect the reality: a typical perforation was produced in the apical part of the buccal canal. Here the mechanical instrumentation did not follow the curvature of the root canal at all.

Even if the ledge on the palatal canal had not been formed (the area marked Ld), the continuation of the mechanical treatment would have encountered a serious difficulty towards the apical in the area where the perforation started. Here a new and even more severe change of root canal direction (127 vs. $\left.147^{\circ}\right)$ would have been difficult to negotiate with medium-thickness and inflexible files.

After the very large thickness of the mechanically instrumented area on the palatal canal (ERSC) it can be seen how big was the calibre of the file that was worked with, when the ledge was created.
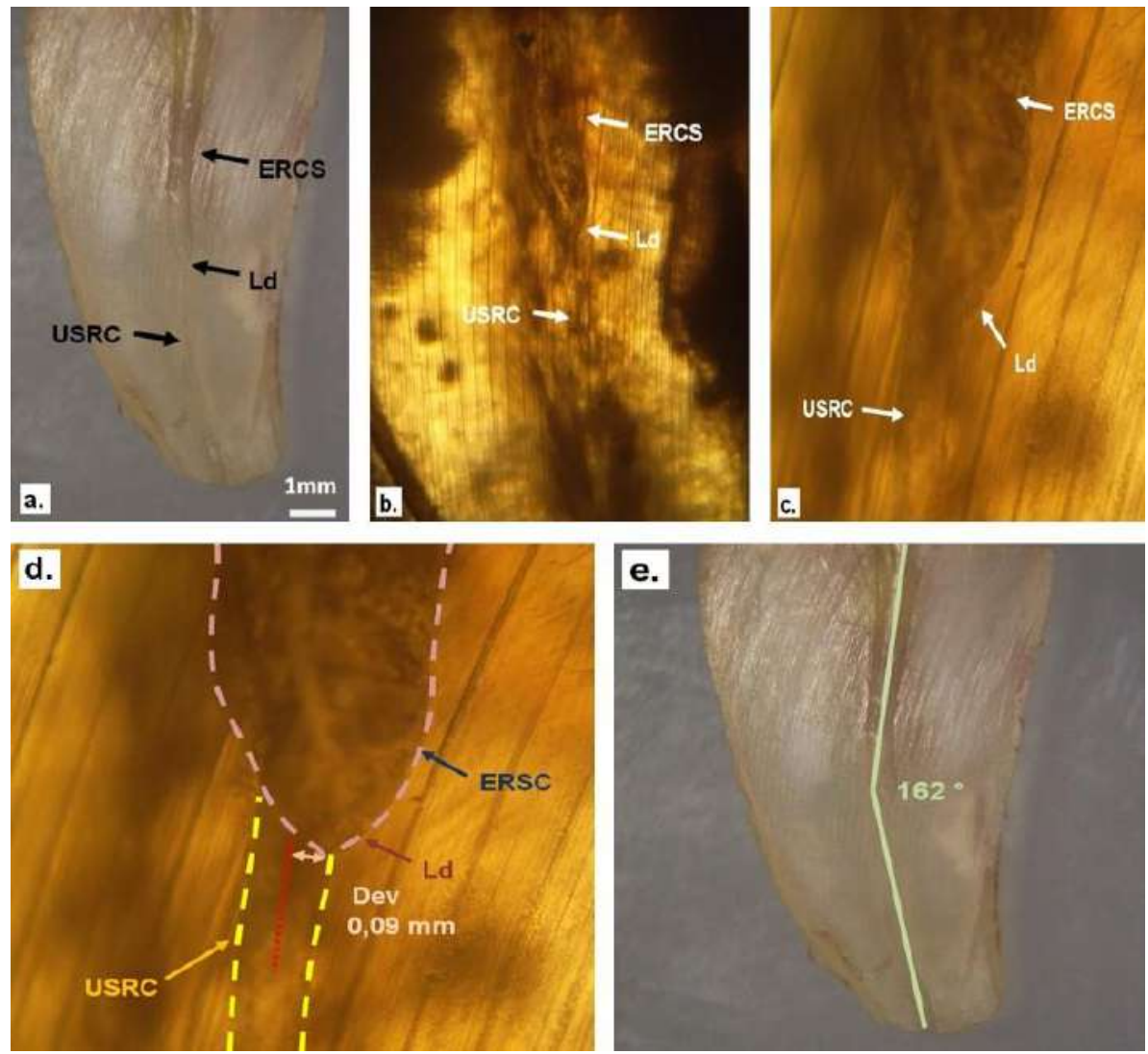

Figure 3. Lateral mandibular incisor (3.2) - Case 2 - Tooth Sample 03 a) Stereomicroscopy image in reflected light (10x); b) Light microscopy image transmitted with the ledge $(30 \mathrm{x})$; c) Detail image of light microscopy transmitted with the ledge $(100 x)$; d) Detail image of light microscopy transmitted with the ledge on which the main aspects of interest are outlined (100x); e) Stereomicroscopy image in reflected light from point a. used for marking and calculating the angle of curvature of the root canal $(10 x)$. Abbreviations used: Ledge $=$ Ld; Enlarged root canal segment $=$ ERCS; Uninstrumented segment of the root canal $=$ USRC; Dev=deviation .

Case no. 2: Lateral mandibular incisor with a ledge formed on the internal curvature of the root canal-In the case of this root of the lateral mandibular incisor, strangely the ledge was formed not towards the outside of the canal curvature as is usually the case, but towards the inside. This aspect is best seen in Fig. $3 \mathrm{c}$ and $3 \mathrm{~d}$, where the higher degree of magnification allows the visualization of this. The relatively small curvature of the root could in no way be considered a favourable factor. We explain this situation by the fact that the file that generated the ledge was precurved by the clinician when meeting a resistance to advance, during the mechanical treatment of the canal, but this precurving was done exaggeratedly and only in the tip area of the file. Thus, the file dug into the root dentin predominantly towards the inner part of the curve. Energetic forward-retracting movements were probably performed. It is also quite probable that thicker and thicker files starting with thin ones were not used for 
instrumentation. Practically, with the thick file that was worked, it was possible to advance by forcing the instrumentation only up to the level where the ledge was subsequently created after the instrumentation was continued with a file of too large calibre (possibly the same) wrongly precurved. It is observed that the direction in which the tip of the precurved file was oriented was the correct one, the direction in which the file dug into the root dentin was consistent with the general curvature of the root, but the degree of pre-curvature was totally erroneous.

It is very likely that this ledge could be easily overcome by using a much thinner file that is less precurved. The excessively large thickness of the last file used, in relation to the thickness of the uninstrumented part of the root canal in the portion immediately following the apical to the file, is clear from Fig. 3d., where the markings delimit the enlarged portion of the root canal (ERSC) and the uninstrumented portion of the root canal (USRC).
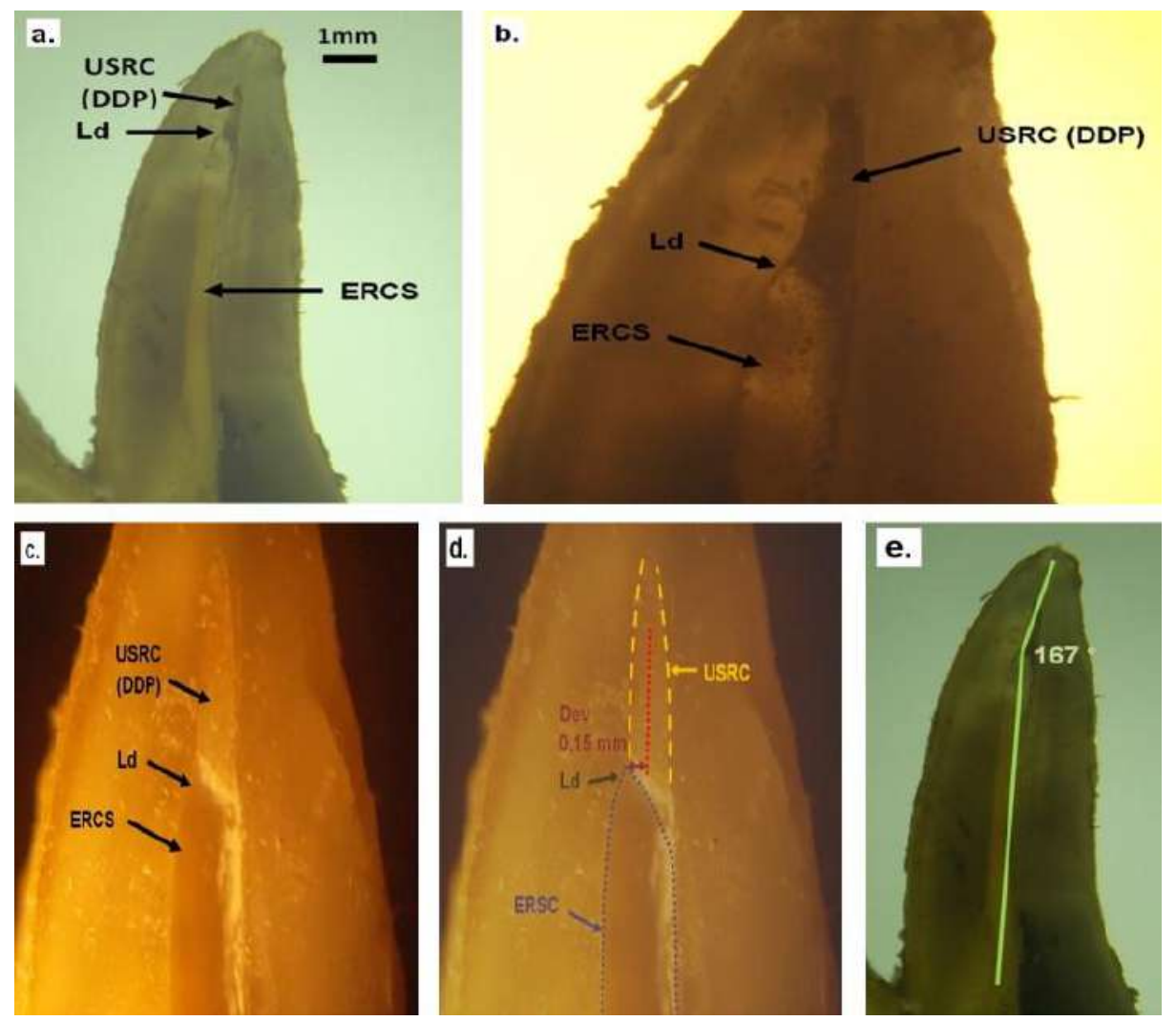

Figure 4. Distobuccal root of a second maxillary molar (2.7)- Case 3 - Tooth Sample 04. a) Stereomicroscopy image in reflected light $(10 \mathrm{x})$; b) Light microscopy image transmitted with the ledge (30x); c) Detail image of light microscopy transmitted with the ledge $(100 x)$; d) Detail image of light microscopy transmitted with the ledge on which the main aspects of interest are outlined (100x); e) Stereomicroscopy image in reflected light from point a. used for marking and calculating the angle of curvature of the root canal $(10 \mathrm{x})$.

Abbreviations used: Ledge $=$ Ld; Enlarged root canal segment =ERCS; Uninstrumented segment of the root canal = USRC; $\mathrm{DDP}=$ dentine debris plug; Dev=deviation.

Case no. 3. Second maxillary molar with a ledge present on the external curvature of the disto-buccal canal. The ledge was identified on the disto-buccal root of a maxillary second molar (tooth 2.7). It is observed that the ledge is formed towards the outer part of the canal curvature, as is usually the case. Fig. $4 \mathrm{~b}$ and $4 \mathrm{c}$ show in the unprepared part of the canal the accumulation of a dentinal detritus in the form of a plug. Although the curvature of the canal in the apical part of the root is not large $\left(167^{\circ}\right)$, it seems that the formation of this ledge was favoured by a second curvature of the root in a plane relatively perpendicular to the sectioning section, namely palatal (oral). This is also verifiable by images $4 \mathrm{a}$ and $4 \mathrm{~b}$, on which it is observed that the unprepared portion of the canal could not be completely captured, up to the apex, in the sectioning plan planned and made by us. In these images, the uninstrumented portion of the canal appears in the form of a spearhead, representing only the part that was captured from the recurved part and distally but also palatally (orally).Thus, when the ledge was formed, the endodontic file used deviated from the direction of the canal, digging in the root dentin simultaneously to the mesial to the buccal (vestibular). 

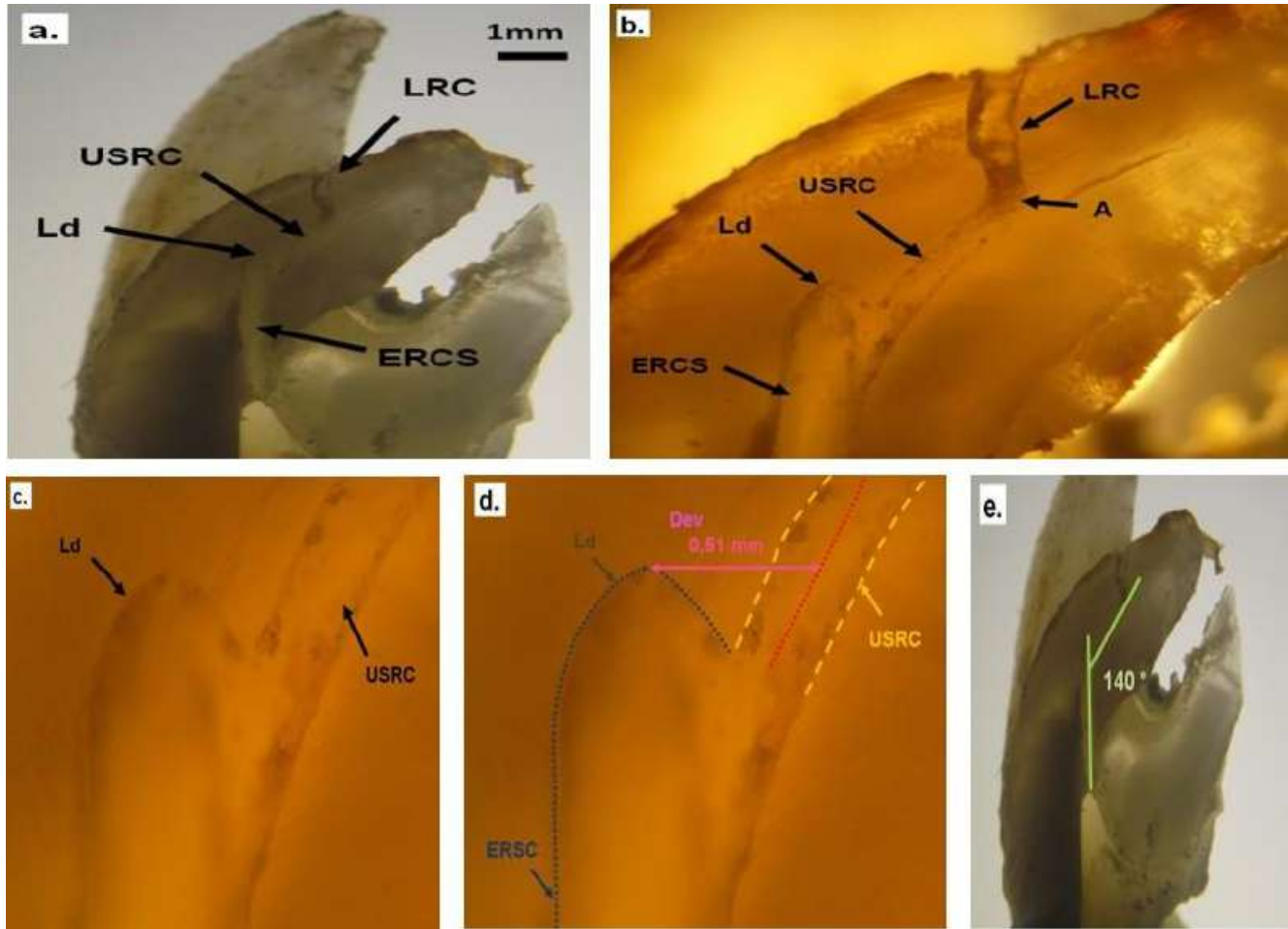

Figure 5. Mesiobuccal root of a first maxillary molar (1.6)- Case 4 - Tooth Sample 05. a) Stereomicroscopy image in reflected light (10x); b) Light microscopy image transmitted with the ledge (30x); c) Detail image of light microscopy transmitted with the ledge $(100 x)$; d) Detail image of light microscopy transmitted with the ledge on which the main aspects of interest are outlined (100x); e) Stereomicroscopy image in reflected light from point a. used for marking and calculating the angle of curvature of the root canal $(10 x)$. Abbreviations used: Ledge $=$ Ld; Enlarged root canal segment = ERCS; Uninstrumented segment of the root canal $=$ USRC; LRC = lateral root canal; Dev = deviation.

Case no. 4. First maxillary molar with a ledge formed on the mesio-buccal root-The ledge was identified on the mesio-buccal root of a first maxillary molar. Both microscopically and macroscopically, the accentuated curvature of the root was obvious. The mechanical instrumentation of the canal led to a preparation that did not negotiate the curvature of the canal at all. Starting from the pulpal chamber to the apical, the preparation is almost perfectly straight (Fig. 5a). The tip of the last files used for the mechanical treatment dug much to the side of the remaining unprepared part of the root canal (Ld in Fig. 5b). The unprepared portion of the root canal (USRC) appears to terminate somewhere on the side of the root (Fig. 5b). In fact, at the point marked ' $A$ ' in Fig. $5 b$ from the remaining unprepared portion of the root canal (USRC) a lateral root canal (LRC) detaches. From this point ' $A$ ' to the apical, the main root canal left unprepared was not captured due to the existence of an additional curve of the root to the distal. This curvature, which I have called 'additional' here, obviously could not be captured by the sectioning plan. Also in point ' $A$ ' where the lateral canal detaches, there are residual residues from the pulp tissue that could not be removed by us by washing under pressure. In Fig. 5c the shape of the ledge can be observed: it does not have the shape of a step but has a shape that corresponds to the tip of the file with which it was acted in the wall of the root canal, instead of acting along it. In Figure 5d the file and the limits of the unprepared portion of the root canal are clearly marked. The size of the deviation is also marked (distance from the top of the ledge to the axis of the unprepared part of the root canal). This deviation of $0.51 \mathrm{~mm}$ is the highest value found in this study.
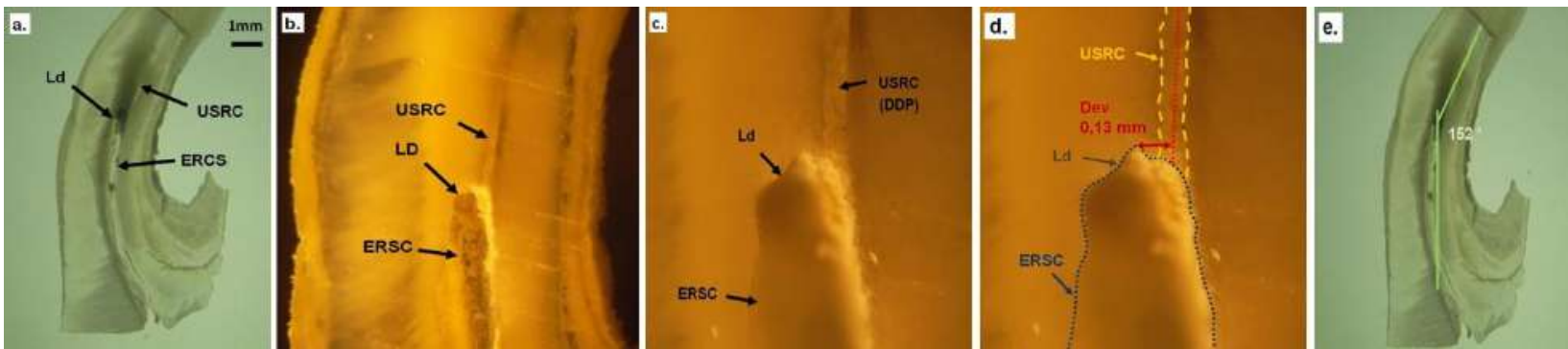

Figure 6. Mesiobuccal root of a first maxillary molar (2.6)- Case 5 - Tooth Sample 07. a) Stereomicroscopy image in reflected light $(10 \mathrm{x})$; b) Light microscopy image transmitted with the ledge (30x); c) Detail image of light microscopy transmitted with the ledge (100x); d) Detail image of light microscopy transmitted with the ledge on which the main aspects 
of interest are outlined (100x); e) Stereomicroscopy image in reflected light from point a. used for marking and calculating the angle of curvature of the root canal (10x).

Abbreviations used:Ledge $=$ Ld; Enlarged root canal segment $=$ ERCS; Uninstrumented segment of the root canal = USRC; $\mathrm{DDP}=$ dentine debris plug; Dev=deviation.

Case no. 5- First maxillary molar with a ledge located on the external curvature of the mesio-buccal canal-In the case of this mesio-buccal root of this first maxillary molar, a ledge located approximately halfway to the root was identified. It is located towards the outside of the canal curvature, as is typically the case (Fig. 6a). We appreciate the curvature of the root and implicitly of the canal to be moderate $\left(140^{\circ}\right)$. In Fig. 6b. it is observed how wide the prepared portion of the root canal (ERSC) is compared to the remaining unprepared portion (USRC). This difference is unacceptably large, given that a possible exceeding of such a ledge could not be made with a file as thick as would correspond to the size of the prepared part of the canal. Also in this image in the prepared part of the root canal (ERSC) is seen the remaining sealer, which due to the very high adhesiveness could not be completely removed. In Fig. $6 \mathrm{c}$ the shape of the ledge is most clearly observed. In this case, too, the ledge does not have a flat shape, but is rather corresponding to the tip of the file with which the prepared portion was last widened. In Figure 6d, the shape of the prepared part of the canal in the ledge area is clearly marked. Compared to other cases, it is much more irregular. The existence of this form could be explained by the high pressure applied to the endodontic files in an attempt to exceed the ledge; this pressure led to the deformation of the tip part of the file and implicitly to a more irregular excavation in the root dentin, at an immediately coronal level compared to the file. Large, active pointed files were probably used.

Although the deviation from the axis of the unprepared part of the root canal is not large, given how wide the ledge is, overcoming it by finding with the tip of a very thin file the unprepared portion of the root canal would have been extremely difficult to do, especially since, as shown in Fig. 6c, the incipient portion of the unprepared part of the canal is blocked with a dentinal detritus plug (DDP) of remarkable compactness. These could not be removed by pressure washing performed by us.
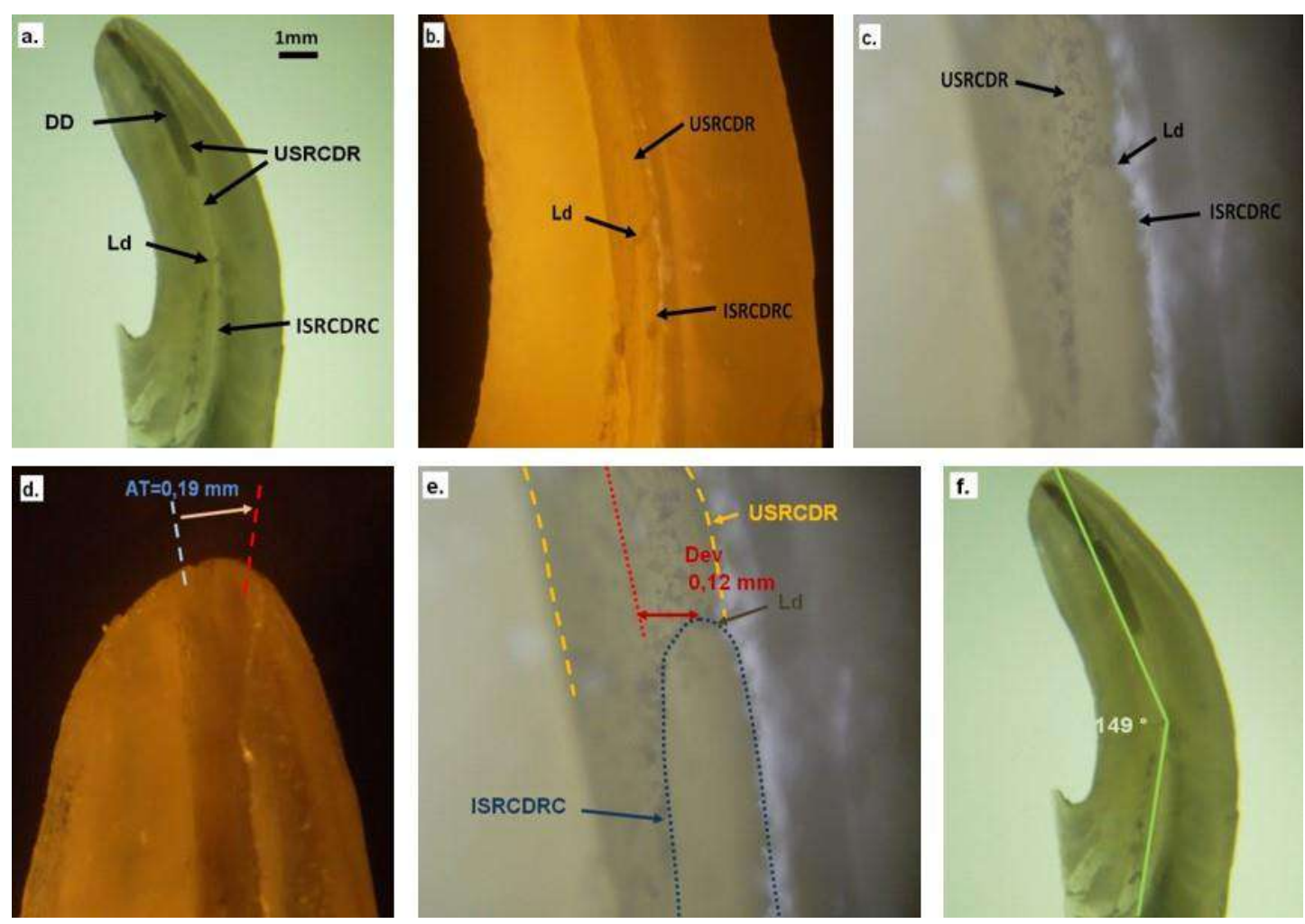

Figure 7. Mesiobuccal root of a first maxillary molar (1.6)-Case 6 - Tooth Sample 08. a) Stereomicroscopy image in transmitted light (10x); b) Microscopy image in light reflected with the ledge (30x); c) Detail image of light microscopy reflected with the ledge (100x); d) Detail image of light microscopy reflected with apical transposition performed at the first mechanical root canal treatment (100x); e) Detail image of light microscopy reflected with the ledge on which the main aspects of interest are outlined $(100 x)$; f) Light stereomicroscopy image transmitted from point a. used for marking and calculating the angle of curvature of the root canal (10x). Abbreviations used: Ledge $=\mathrm{Ld}$; Instrumented segment of the radicular canal during root canal retreatment = ISRCDRCR; Uninstrumented segment of the radicular canal during root canal retreatment $=$ USRCDRCR; Apical transposition $=$ AT; DD=dentine debris; Dev=deviation. 
Case 6 is a special case in which a ledge appeared. This one was formed on the mesio-buccal root of a first maxillary molar during the retreatment and not at the first mechanical instrumentation as happens in most cases. In this case on the root section we distinguish the instrumented portion of the root canal at the resumption of endodontic treatment (ISRCDRCR) and the uninstrumented portion of the root canal at the retreatment (USRCDRCR) (7a and 7b). The ledge formed is most clearly seen in Fig. 7c and represents an excavation in the wall of the first mechanical preparation of the canal. It can also be seen in this photo that the width of the first preparation is larger (almost double the width of the second mechanical preparation).

Most likely, the advanced degree of consolidation of the first root canal filling did not make it possible for the endodontic file, during the retreatment, to advance through the first filling, so it acted more easily laterally than it in the root dentin. This was also favoured by the change of the canal direction at this level. From this microscopic analysis it is quite obvious that when resuming the canal treatment, almost no attempt was made to dissolve the gutta-percha in the first filling.

The first endodontic treatment was unsuccessful. As proof we bring the image from the apex of an apical transposition, which we evaluated, by microscopic micrometry techniques to be approximately $0.19 \mathrm{~mm}$. This was probably also the reason why the canal treatment was resumed. In addition, as shown in image 7a (stereomicroscopy overview) $\mathrm{m}$ in the apical portion of the canal there is a portion rich in artificially cemented dentinal detritus (DD) by means of the sealer used in the first canal filling. This dentinal detritus could not be removed by our pressure washings. As mentioned in the introduction, no attempt was made to remove such accumulations of detritus with metal instruments so as not to alter the characteristics of the endodontic space remaining from the preparations made in vivo and whose capture was in fact an objective in this study.

In Table 1, we grouped the data on root canal curves for all studied roots and deviations encountered for all endodontic ledges that were analysed.

Table 1. The main characteristics of the root canals on the studied roots and of the ledges identified on them

\begin{tabular}{|c|c|c|c|c|c|c|}
\hline $\begin{array}{c}\text { Tooth } \\
\text { sample no. }\end{array}$ & 1 & \multicolumn{2}{|c|}{2} & 3 & 4 & 5 \\
\hline Tooth & 1.4 & \multicolumn{2}{|c|}{2.2} & 2.3 & 2.7 & 1.6 \\
\hline Root & $\begin{array}{l}\text { single root with two } \\
\text { canals (Weine type II } \\
\text { configuration) }\end{array}$ & \multicolumn{2}{|c|}{ single root with one canal } & $\begin{array}{l}\text { single root with } \\
\text { one canal }\end{array}$ & $\begin{array}{l}\text { disto-buccal root } \\
\text { with one canal }\end{array}$ & $\begin{array}{l}\text { mesio-buccal root } \\
\text { with one canal }\end{array}$ \\
\hline Ledge location & $\begin{array}{c}\text { lingual canal - } \\
\text { middle third }\end{array}$ & \multicolumn{2}{|c|}{ apical third } & middle third & apical third & Middle third \\
\hline $\begin{array}{c}\text { Main curvature } \\
\text { (diection and degree) }\end{array}$ & $\begin{array}{c}147^{0} \\
\text { (towards buccal) }\end{array}$ & \multicolumn{2}{|c|}{$\begin{array}{c}153^{\circ} \\
\text { (towards distal) }\end{array}$} & $\begin{array}{c}162^{0} \\
\text { (towards distal) }\end{array}$ & $\begin{array}{c}167^{0} \\
\text { (towards distal) }\end{array}$ & $\begin{array}{c}140^{\circ} \\
\text { (towards lingual) }\end{array}$ \\
\hline $\begin{array}{l}\text { Secondary curvature } \\
\text { (direction and degree) }\end{array}$ & ( & \multicolumn{2}{|c|}{ (2) } & ( & $\begin{array}{c}\approx 160^{0} \\
\text { (towards oral) }\end{array}$ & $\begin{array}{c}\approx 155^{0} \\
\text { (towards distal) }\end{array}$ \\
\hline Deviation $(\mathrm{mm})$ & 0.11 & \multicolumn{2}{|c|}{0.15} & 0.09 & 0.15 & 0.51 \\
\hline $\begin{array}{c}\text { Tooth } \\
\text { sample no. }\end{array}$ & 6 & 7 & 8 & 9 & 10 & 11 \\
\hline Tooth & 3.5 & 2.6 & 1.6 & 3.6 & 1.7 & 3.3 \\
\hline Root & $\begin{array}{l}\text { single root with one } \\
\text { canal }\end{array}$ & $\begin{array}{c}\text { mesio-buccal } \\
\text { root with one } \\
\text { canal } \\
\end{array}$ & $\begin{array}{c}\text { mesio-buccal } \\
\text { root with one } \\
\text { canal } \\
\end{array}$ & $\begin{array}{c}\text { Distal root with } \\
\text { one canal }\end{array}$ & $\begin{array}{l}\text { disto-buccal root } \\
\text { with one canal }\end{array}$ & $\begin{array}{l}\text { single root with } \\
\text { one canal }\end{array}$ \\
\hline Ledge location & apical third & middle third & middle third & apical third & middle third & apical third \\
\hline $\begin{array}{c}\text { Main curvature } \\
\text { (diection and degree) }\end{array}$ & $\begin{array}{c}160^{\circ} \\
\text { (towards distal) }\end{array}$ & $\begin{array}{c}152^{0} \\
\text { (towards distal) }\end{array}$ & $\begin{array}{c}149^{0} \\
\text { (towards distal) }\end{array}$ & $\begin{array}{c}143^{0} \\
\text { (towards distal) }\end{array}$ & $\begin{array}{c}156^{0} \\
\text { (towards distal) }\end{array}$ & $\begin{array}{c}157^{0} \\
\text { (towards distal) }\end{array}$ \\
\hline $\begin{array}{l}\text { Secondary curvature } \\
\text { (diection and degree) }\end{array}$ & ( & $\begin{array}{c}\approx 165^{\circ} \\
\text { (towards } \\
\text { lingual) } \\
\end{array}$ & $\begin{array}{c}\approx 170^{\circ} \\
\text { (towards } \\
\text { lingual) } \\
\end{array}$ & 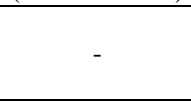 & $\begin{array}{c}\approx 170^{\circ} \\
\text { (towards lingual) }\end{array}$ & 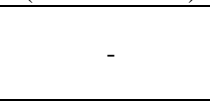 \\
\hline Deviation $(\mathrm{mm})$ & 0.12 & 0.13 & 0.12 & 0.14 & 0.16 & 0.12 \\
\hline
\end{tabular}

\section{Conclusions}

The shape of the ledges - is not the step shape (as the English term suggests) but has a shape that corresponds to the negative of the tip of the file with which it was created. In 3D the shape is somewhat conical. In almost all the cases we investigated, we identified a ledge's peak. The ledges do not always appear on the outer curvature of the canals as classically described. We also encountered an exception.
The appearance of a ledge on the internal curvature is due to its improper pre-curvature and its actuation by pushing and retracting inadvertently;

The largest deviations are associated with the highest degrees of curvature of the roots (tooth sample 5). Note that in the case of the values in degrees used by us, a decrease in value actually implies an increase in the degree of curvature. Relatively small deviations in the sectioning plane give the feeling that the respective ledge would be 
easily overcome by a re-instrumentation. The seemingly minor aspects in the section plane corresponding to the main curve are in fact complicated by the presence of a second curve which is in a different plane from that of the main curve (see tooth sample 7 and 8).

One can speak of an association of the production of ledges with that of the production of false paths. Practically, a false root pathway results from the continuation of penetration into the root dentin starting from a ledge. If, after the formation of a ledge, the endodontic instrument is insisted on the wrong direction and in that place the root curvature is accentuated, an exit can be reached in the periodontal space.

\section{Acknowledgments} author.

All the authors have equal contributions as the first

\section{References}

1. JAFARZADEH, HAMID et al. Ledge Formation: Review of a Great Challenge in Endodontics. Journal of Endodontics 2007, 33(10): 1155-1162.

2. SCARLATESCU SA, SUCIU I, GHEORGHIU IM, PERLEA P, DIMITRIU D, IACOMI LD, DAINA LUCIA L, PIRCALABIORU GG, BARTOK R,
STEFA DS. Comparative analysis of the temporary obturation materials applied to M-O-D cavities in endodontically treated teeth. Rom Biotechnol Lett. 2019; 24(2): 280-285. DOI: $10.25083 / \mathrm{rbl} / 24.2 / 280.285$

3. HÜLSMANN M., SCHÄFER E. Problems in root canal preparation. In: Problems in Endodontics: Etiology, Diagnosis and Treatment. 1st ed. Germany: Quintessence Publishing; 2009, pp. 209-251.

4. KAPALAS A, LAMBRIANIDIS T. Factors associated with root canal ledging during instrumentation. Endod Dent Traumatol 2000; Department of Endodontology, School of Dentistry, 16: 229-231.

5. LAMBRIANIDIS, T. Ledging and blockage of root canals during canal preparation: causes, recognition, prevention, management, and outcomes. Endodontic Topics, 2006; 15(1), 56-74.

6. ELEFTHERIADIS GI, LAMBRIANIDIS TP. Technical quality of root canal treatment and detection of iatrogenic errors in an undergraduate dental clinic. Int Endod J. 2005; 38:725-34.

7. KHANNA R, HANDA A, VIRK RK, VGHAI D, HANDA RS, GOEL A. Clinical and radiographic evaluation of procedural errors during preparation of curved root canals with hand and rotary instruments: A randomized clinical study. Contemp Clin Dent 2017; 8:411-5. 